\title{
Article
}

\section{Peripheral preview abolishes N170 face- sensitivity at fixation: Using fixation- related potentials to investigate dynamic face processing}

de Lissa, Peter, McArthur, Genevieve, Hawelka, Stefan, Palermo, Romina, Mahajan, Yatin, Degno, Federica and Hutzler, Florian

Available at http://clok.uclan.ac.uk/30342/

de Lissa, Peter, McArthur, Genevieve, Hawelka, Stefan, Palermo, Romina, Mahajan, Yatin, Degno, Federica ORCID: 0000-0002-9621-9968 and Hutzler, Florian (2019) Peripheral preview abolishes N170 face-sensitivity at fixation: Using fixation-related potentials to investigate dynamic face processing. Visual Cognition, 27 (9-10). pp. 740-759. ISSN 1350-6285

It is advisable to refer to the publisher's version if you intend to cite from the work. http://dx.doi.org/10.1080/13506285.2019.1676855

For more information about UCLan's research in this area go to http://www.uclan.ac.uk/researchgroups/ and search for <name of research Group>.

For information about Research generally at UCLan please go to http://www.uclan.ac.uk/research/

All outputs in CLoK are protected by Intellectual Property Rights law, including Copyright law. Copyright, IPR and Moral Rights for the works on this site are retained by the individual authors and/or other copyright owners. Terms and conditions for use of this material are defined in the policies page. 
This is an Accepted Manuscript of an article published by Taylor \& Francis in Visual Cognition.

This paper is not the copy of record and may not exactly replicate the final, authoritative version of the article. Please do not copy or cite without authors' permission. The final article will be available, upon publication, at the following link: https://doi.org/10.1080/13506285.2019.1676855 


\section{Peripheral preview abolishes N170 face-sensitivity at fixation: Using}

\section{fixation-related potentials to investigate dynamic face processing}

Peter de Lissa ${ }^{1,2 *}$, Genevieve McArthur ${ }^{2}$, Stefan Hawelka ${ }^{3}$, Romina Palermo ${ }^{4}$, Yatin Mahajan $^{5}$, Federica Degno ${ }^{6}$, and Florian Hutzler ${ }^{3}$

1. iBMLab, Department of Psychology, University of Fribourg, Fribourg, Switzerland

2. Department of Cognitive Science, Macquarie University, Sydney, Australia

3. Centre for Cognitive Neuroscience, Salzburg University, Salzburg, Austria

4. School of Psychological Science, University of Western Australia, Perth, Australia

5. The MARCS Institute, University of Western Sydney, Australia

6. School of Psychology, University of Central Lancashire, Preston, United Kingdom

* Corresponding author

Peter de Lissa

University of Fribourg

Faucigny 2

1700 Fribourg

Switzerland

Email: peter.delissa@unifr.ch 


\title{
Peripheral preview abolishes N170 face-sensitivity at fixation: Using fixation-related potentials to investigate dynamic face processing
}

\begin{abstract}
The N170 event-related potential (ERP) has been found to be consistently larger in response to the presentation of faces than to other objects. It is not yet clear if this face-sensitive N170 is elicited by the initial detection of a face, or whether it perseveres during further exploration of a face. To investigate this question, the current study utilised the Fixation-Related Potentials (FRP) technique in two experiments, time-locking brain potentials to the presentation of images of faces and objects (watches) in participants' peripheral vision, and to their first fixations on the same images. Experiment 1 found that a face-sensitive N170 was elicited by the onset of images but not by the onset of the first fixation on those images, and that face inversion did not modulate N170 beyond presentation. Experiment 2 found that when the structure in the peripheral preview was disrupted by phase-scrambling the images, and when those scrambled images were unscrambled during the orienting saccade to the stimuli, a face-sensitive N170 was observed at the onset of fixation rather than the onset of image presentation. Interestingly, N170 amplitudes for both faces and objects were significantly enhanced when the peripheral preview was phase-scrambled, suggesting that the N170 in part reflects a category-detection process that is elicited once when an image structure is viewed. These results indicate that neural processing during fixations will be significantly modulated when they are immediately preceded by peripheral previews, and is not specific to faces.
\end{abstract}

Keywords: 
Faces, N170, ERP, FRP, Fixation 
Most of our visual perception occurs through natural fixations, where we are often able to control the timing and the targets of our visual processing. Our peripheral field of view allows us to perceive and process these targets, and subsequently direct our gaze towards them to process them with greater acuity through our foveal vision (Strasburger, Rentschler, \& Juettner, 2001 for review). One of the most salient targets for our gaze is the human face, which tends to attract our attention more than other visual stimuli (Langton et al., 2008; Lavie et al., 2003; Yarbus, 1967; Palermo \& Rhodes, 2007, for review). The preferential processing of faces in our periphery has been demonstrated by the significant speed advantage we have when making saccades towards images of faces than to other familiar objects such as vehicles (Crouzet et al., 2010; Guyader et al., 2017; Kauffmann et al., 2019) or houses (Mares et al., 2016).

Neuroimaging studies have consistently found that faces can be differentiated from other objects very early in the visual processing stream. In event-related potential (ERP) studies, this can be seen with a negative peak, the N170, at around 170 milliseconds (ms) over occipitotemporal areas that is (1) larger to faces than other objects (e.g., watches, cars, or houses; Bentin et al., 1996; Eimer, 1998; Rossion \& Jacques, 2007; although cf Thierry, 2007); (2) later and larger to inverted faces than upright faces (Itier \& Taylor, 2004; Itier et al., 2006; Linkenkaer-Hansen et al. 1998; Rossion et al., 2000); and (3) elicited by faces presented in either the centre of a visual field or the periphery (when spatially scaled to account for eccentricity effects) (Kovács et al., 2017; Rousselet et al., 2005). Of note is the insensitivity of the N170 to face identity in typical ERP paradigms, which has led to the suggestion that this early peak reflects a structural encoding stage of processing of facial features and configuration that are used in later stages of processing such as recognition (Bentin et al., 1996; Bentin \& Deouell, 2000; Eimer, 2000). While this interpretation follows from the model of structural encoding for faces initially outlined by Bruce and Young (1986), 
the N170 is also considered to reflect a face detection process (George et al., 2005; Sagiv \& Bentin, 2001), which is not explicitly part of Bruce and Young's model (Rossion \& Jacques, 2010 for review). The relationship between the N170 and behavioural perception of a face was highlighted by the results of George et al. (2005), who found that Mooney faces that had been behaviourally detected as faces elicited larger N170 responses than those that had not.

While the patterns of N170 responses to faces and objects have been the focus of much study in the past decades, traditional ERP paradigms do not lend themselves to the investigation of the processing of these stimuli in more complex contexts mirroring our everyday experience, where visual objects are often initially perceived peripherally and subsequently fixated upon. To date, our understanding of the neural processing of faces and objects has mostly involved the analysis of neural responses to the onset of the image, presented for a short period of time (e.g., a photo presented for $200 \mathrm{~ms}$ ). For practical and experimental reasons, traditional N170 ERP paradigms do not readily allow for the analysis of brain activity beyond the initial presentation of an image, and so the fate of faceprocessing beyond this presentation-related processing is as yet unknown. Studies have shown that the N170 is elicited by faces presented in the periphery (Kovács et al., 2017; 2017; Rousselet et al., 2005), and that people tend to be significantly faster to make saccades to faces than to other objects when presented outside of central vision (Crouzet et al., 2010; Guyader et al., 2017; Kauffmann et al., 2019; Mares et al., 2016). However, it is not yet clear how the peripheral processing of a face (or other object) modulates processing when the images are subsequently fixated upon after this detection occurs. As part of a mechanism reflecting early detection and encoding of faces, an N170 elicited at presentation may not be activated by a subsequent fixation made on the image, as the processes would already be completed by the time of fixation. The consequences of a peripheral preview for processing at a subsequent fixation may therefore have many implications beyond face processing, 
where visual objects may be perceived before a fixation is made to them, such as in reading or in visual search paradigms.

Although the traditional ERP approach is limited in its ability to measure neural activity beyond the initial presentation, an approach that is well-suited to investigate the influence of peripheral previews on subsequent fixation-related face processing is the Fixation-Related Potentials (FRP) paradigm. By co-registering EEG and eye-position recordings, traditional ERPs can be recorded during the initial peripheral presentation of stimuli, and FRPs may be formed by time-locking the EEG to the onset of initial fixations made to the stimuli. So far, the FRP technique has offered a flexible approach to investigate how the brain processes both complex scenes and stimuli, and it applied to object identification (Rama \& Baccino, 2010), free exploration (Devillez, Guyader, \& GuérinDugué, 2015; Graupner et al., 2007; Kamienkowski et al., 2012; Ossandón et al., 2010), foveal/parafoveal wording reading (Baccino \& Manunta, 2005; Degno et al, 2019; Dimigen, Kliegl, \& Sommer, 2012; Hutzler et al., 2007; Hutzler et al., 2013; Kornrumpf et al., 2016; Lopez-Perez et al., 2016; Niefind \& Dimigen, 2016), and attentional shifts (Huber-Huber et al., 2016). The paradigm has also been extended to investigate the processing of faces within complex scenes (Kaunitz et al., 2014), emotionally-salient scenes involving faces and other objects (Guérin-Dugué et al., 2018; Simola et al., 2015; Soto et al., 2018), and the effect face and gaze detection has on saccadic orienting to faces and other objects (Mares et al., 2016).

Our poor understanding of the effect of peripheral preview on subsequent fixations is particularly relevant for these latter studies. Whereas faces have been found to elicit larger N170 amplitudes than other objects in traditional ERP paradigms, fixations made on these images might not elicit face-sensitivity if a peripheral preview of the images had been available to the participant beforehand. Additionally, peripheral preview effects may affect stimuli differently when normal processing is disrupted. When faces are inverted they tend to 
be more difficult to recognise or make other judgements compared to when they are upright (McKone \& Yovel, 2009; Richler et al., 2011; Sergent, 1984; Tanaka \& Farah, 1993; Tanaka \& Simonyi, 2016 for review). Considered to reflect a disruption of the holistic processing of faces (the processing of face features and their spatial-relations as a united whole), face inversion has also been found to consistently lead to an increase (and delay) in the N170 amplitude (Itier \& Taylor, 2004; Itier et al., 2006; Linkenkaer-Hansen et al. 1998; Rossion et al., 2000). In consideration of how peripheral processing of faces might affect processing when they are fixated, we are yet to have a clear idea of whether face inversion has effects beyond the enhancement of $\mathrm{N} 170$ at presentation, or whether the disruption of normal face processing leads to further modulation in a subsequent fixation.

To address these questions, we measured N170 responses in two separate experiments designed to investigate how peripheral preview modulates subsequent fixation-related activity. Experiment 1 investigated the neural processing of faces during peripheral preview and subsequent fixation by comparing the N170 elicited by faces and objects, and the effects of face inversion. A reliable face-sensitive N170 was observed in response to peripheral presentation but not in the first fixation made on the images. Additionally, a larger N170 amplitude to inverted faces than upright faces was observed at the peripheral presentation, but there was no effect of inversion at fixation. Experiment 2 tested whether the absence of a face-sensitive N170 peak at fixation was specifically related to the structural-integrity of the preceding peripheral preview. Preventing peripheral preview of a face by phase-scrambling the preview images led to a significant face-sensitivity at first fixation, and significant enhancement of N170 responses for both face and watch stimuli. These results suggest that the N170 brain potential indexed the processing of the image structures in the current study, and may therefore reflect a categorical detection process that is nevertheless sensitive (although not specific) to faces. 


\section{Experiment 1}

\section{Method}

Experiment 1 investigated face sensitivity, and the effects of peripheral preview on FRPs in a subsequent fixation. Upright and inverted faces and wrist-watches were presented in participants' right periphery, and participants were instructed to fixate upon and scan the images after presentation. EEG and eye-position data were recorded, and ERP/FRP epochs were formed relative to the initial presentation (hereafter "Presentation") and onset of subsequent fixation ("Fixation") on the images, respectively.

\section{Ethics}

The Human Ethics Committee at Macquarie University approved the methods and procedures used in this study.

\section{Participants}

Twenty participants were recruited for the experiment. Two were excluded due to excessive noise in the EEG data, and two were excluded due to corrupted and incomplete eye-tracking records. This left complete datasets for sixteen participants (11 females, 14 right-handed), who were aged between 19 and 30 years (mean age $=23.5$ years, $\mathrm{SD}=2.9$ ). All participants had normal or corrected-to-normal vision, and gave their informed consent before participating in the study. Participants were offered \$30AUD for their time.

\section{Stimuli and procedure}

Stimuli comprised upright and inverted faces and watches. Face stimuli consisted of 100 grey-scale images of emotionally neutral Caucasian individuals (50 female, 50 male), cropped within a standard sized oval frame where only the internal face parts were visible (see Figure 1 for example stimuli). The face images were obtained from seven databases: NimStim (Tottenham, et al., 2002), the Karolinska Directed Emotional Faces (KDEF; Lundqvist, et al., 1998), Gur et al. (2002), Computational Vision Archive (courtesy of 
Caltech), the MIT-CBCL (Weyrauch, et al., 2004), the Ekman and Friesen Pictures of Facial Affect (Ekman \& Friesen, 1976), and a set from St Andrews University (courtesy of David Perrett). Watch stimuli were grey-scale images of 50 different wristwatches sourced from the University of Kansas Information and Telecommunication Technology Center database. Watches were chosen because they were familiar objects, were similar in shape to the faces (i.e., oval), and have been used as non-face stimuli in previous ERP studies (e.g., Bentin et al., 2007). Like the face stimuli, each wristwatch was cropped to fit within a standard size oval frame. The mean pixel intensities of the face and watch stimuli were 0.23 (0.03SD) and $0.27(0.03 \mathrm{SD})$, respectively, and the mean root mean square (RMS) contrasts were $0.38(0.04 \mathrm{SD})$ and $0.37(0.03 \mathrm{SD})$ (values normalised to $0-1$ range).

There were 300 trials in total: 100 for upright faces, 100 for inverted faces, 50 trials with upright watches, and 50 trials with inverted watches. A smaller number of trials were used in the watch conditions because 50 trials is an adequate number of trials to create a reliable waveform in adults, and to decrease fatigue in the participants. 100 trials were chosen in the face conditions to allow a greater signal-to-noise ratio in order to capture the less consistently found amplitude face-inversion effects (Bentin et al., 1996). The inverted watch condition was included to provide participants with both upright and inverted examples of both watch and face stimuli, although the inverted watch condition was not related to the task or the analysis.

Stimuli were presented randomly in heterogeneous blocks with short breaks after every 100 trials, on a 19" CRT computer monitor with a refresh rate of $100 \mathrm{~Hz}$ at a distance of $50 \mathrm{~cm}$ from the participant. To ensure that the face and watch stimuli were spatially-scaled to compensate for differences in cortical magnification related to peripheral presentation (Rousselet et al., 2005), each image was 17.4 x 12.7 degrees of visual angle and presented 9.5 degrees of visual angle (centred) to the right of a central fixation cross. 
Participants were fitted with the electroencephalography (EEG) cap and positioned in a head-stabilised eye-tracking chinrest. The EEG was recorded through $30 \mathrm{Ag}-\mathrm{AgCl}$ sintered electrodes embedded in an elastic cap (EasyCap) positioned according to the 10-20 system, running through a Synamps II amplifier at a sampling rate of $1000 \mathrm{~Hz}$, an online band-pass filter of 0.01 to $100 \mathrm{~Hz}$, and a notch filter at $50 \mathrm{~Hz}$. The left and right earlobes were used as online references, and ground was located between the Fz and FPz electrodes. Electrode impedances were kept below $5 \mathrm{k} \Omega$. Ocular movement was recorded with bipolar electrodes placed at the outer canthi, and above and below the left eye. Participants' eye-movements were tracked and recorded with a monocular (right eye) Eyelink 1000 eye-tracker sampling at $1000 \mathrm{~Hz}$. The eye-tracker utilised a 9-point calibration at the beginning of each block of trials. A gaze-contingent fixation cross activated the presentation of each trial, which served as an additional check of eye-tracker calibration for each trial. When the fixation cross was not fixated upon (an area corresponding to a circle of 1 degree of visual angle in radius centred on the cross) within four seconds, a calibration process was automatically entered.

Stimulus presentation was controlled by Experiment Builder software (version 1.6.1). utilising a gaze-contingent white fixation cross in the central-left part of a computer monitor to initiate the presentation of stimuli. Once the participant had fixated on the cross for 150 ms, an upright or inverted face or watch was presented 9.5 degrees of visual angle (centred) to the right of the fixation cross. Participants were then free to move their eyes to the images and scan them freely for $5000 \mathrm{~ms}$. This instruction was given to avoid modulating natural orienting towards the stimuli. Each trial was followed by a "blink now" screen that was displayed for $1500 \mathrm{~ms}$ before the next trial started. The testing session lasted for approximately 1 hour for each participant.

\section{Eye-movement processing and EEG co-registration}


During the trials, the presentation software sent trial-specific serial port codes to both the EEG and eye-tracker recording to mark the onset of the stimulus presentation. These time-stamps were used in the eye-tracking analysis to determine the times of the first fixation made to the images relative to presentation, which were then used as additional time-stamps for the EEG analysis. This allowed the EEG to be time-locked to both the presentation of the stimuli as well as to the first fixation made on the stimuli. Trial-by-trial eye-movement data was analysed through Data Viewer software (1.11.1) for the purpose of time-locking EEG epochs to the initial fixation on the images after presentation, and to ensure that only trials involving appropriate eye-movement behaviour were included in the analysis. Thus, each trial was screened to exclude (1) saccade reaction times (orienting saccades towards the images) which were implausibly quick ( $<100 \mathrm{~ms})$ or too slow (>1000 ms), (2) saccade lengths and fixation landing positions that did not correspond to the presented images, (3) there were any intervening fixations made before the first fixation to the images, and (4) initial stimulus fixations that were too brief $(<150 \mathrm{~ms})$. The initial landing locations from the first fixation to the stimuli are presented for visual purposes in Figure 1, where a typical centre-eye region location can be observed for the initial landing fixation. Thus, the fixations fell on face regions that have been found to elicit the clear face-sensitive N170 in traditional ERP paradigms, where fixation-location might otherwise have modulated neural responses but not abolished this face-sensitivity (de Lissa et al., 2014; Nemrodov et al., 2014). 
a
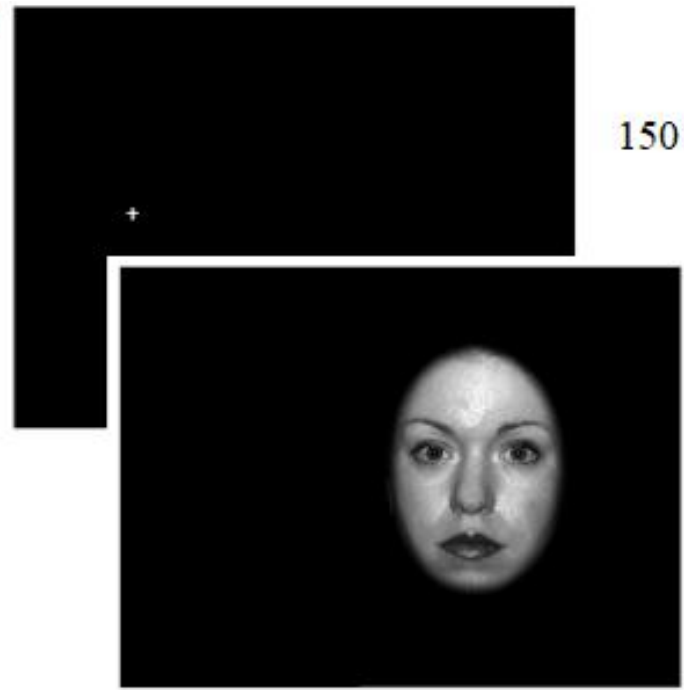

b
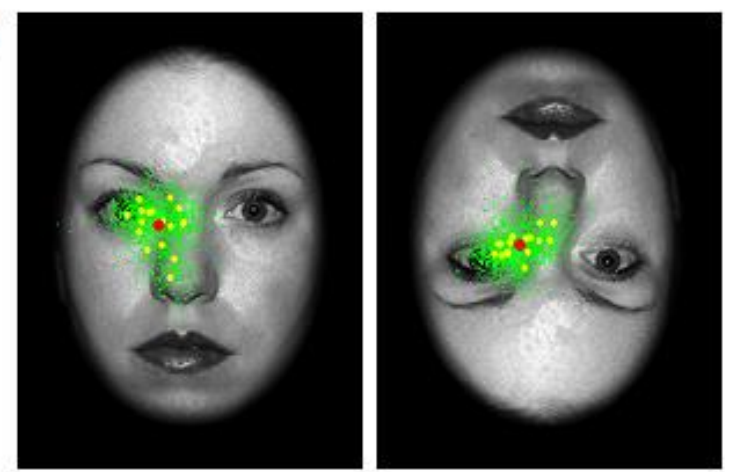

$150 \mathrm{~ms}$

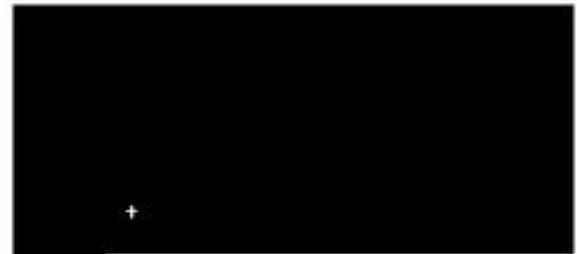

\section{$5000 \mathrm{~ms}$}
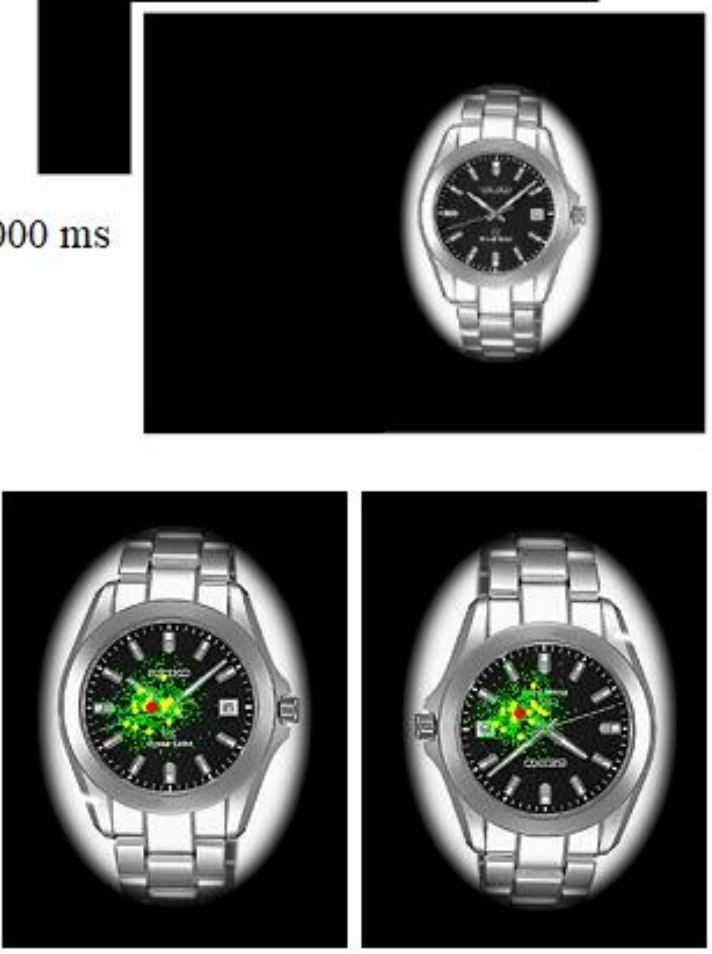

Figure 1. (a) Trial order and (b) examples of upright and inverted face and wristwatch stimuli presented in Experiment 1. Coloured data points illustrate the first fixations made to the images, representing the fixation locations of the group average (red), the subject average (yellow), and individual trials (green). Face image taken from the NimStim Set of Facial Expressions (Tottenham, et al., 2002).

To compare the time taken to fixation on the images after peripheral presentation, fixation latency was analysed with a repeated measures ANOVA, which did not find significant main effects for stimulus type (faces/watches) $[F(1,16)=.19, p=.67]$ or stimulus orientation (upright/inverted) $[F(1,16)=1.54, p=.23]$, nor an interaction between them $[F(1,16)=1.25, p=.28]$

The duration of first fixations made on the images was similarly analysed, revealing a significant main effect of stimulus orientation, where inverted stimuli led to first fixations that were approximately $13 \mathrm{~ms}$ longer than upright stimuli $\left[F(1,16)=9.19, p=.008, \eta^{2}=\right.$ 
.36]. Stimulus type did not significantly modulate first fixation durations $[F(1,16)=1.54, p=$ $.23]$, nor did it interact with stimulus orientation $[F(1,16)=.003, p=.96]$.

Please insert Table 1 about here

\begin{tabular}{ccccc} 
& \multicolumn{2}{c}{ Faces } & \multicolumn{2}{c}{ Watches } \\
\cline { 2 - 5 } & Upright & Inverted & Upright & Inverted \\
\cline { 2 - 5 } $\begin{array}{c}\text { Trials - } \\
\text { Presentation }\end{array}$ & $95(4.2)$ & $96(2.6)$ & $48(1.8)$ & $48(1.9)$ \\
\hline $\begin{array}{c}\text { Trials - } \\
\text { Fixation }\end{array}$ & $97(4)$ & $98(2.1)$ & $49(1.6)$ & $48(1.7)$ \\
\hline $\begin{array}{c}\text { Fixation } \\
\text { latency (ms) }\end{array}$ & $282(32)$ & $287(34)$ & $279(31)$ & $278(31)$ \\
\hline $\begin{array}{c}\text { Fixation } \\
\text { duration (ms) }\end{array}$ & $210(71)$ & $223(78)$ & $214(65)$ & $227(72)$
\end{tabular}

Table 1. Mean trial numbers, fixation latencies (period between the stimulus presentation and subsequent fixation) and durations of first fixations on the images for all conditions in Experiment 1 in milliseconds (standard deviation in brackets).

\section{EEG and FRP processing}

The EEG data was analysed offline via Neuroscan 4.3 and EEGLAB 11.0.4.3b

software. Each EEG channel was filtered with a band-pass of $0.1-30 \mathrm{~Hz}$ with $12 \mathrm{~dB} /$ octave roll-off. Bad or excessively noisy channels were visually identified, removed, and interpolated. Epochs of 2000 ms lengths were formed, time-locked to the stimulus presentation, which encompassed the time range of the presentation and at least two subsequent fixations. The distortions from ocular artefacts (blink and saccadic activity) were identified, isolated and removed from the epoched EEG data in EEGLAB through independent component analysis (ICA) (Delorme \& Makeig, 2004). The resulting EEG data was re-referenced to the common-average of all scalp electrodes, and two new epochs (-100 to $400 \mathrm{~ms}$ ) were formed: A presentation-related potential time-locked to the time of stimulus presentation in the periphery and a first-fixation-related potential time-locked to the first fixation made on the stimuli after presentation. The presentation-related epochs were baseline corrected to the average of the $100 \mathrm{~ms}$ interval preceding stimulus presentation. The FRPs 
formed by the first fixation on the images were baseline corrected to the average activity immediately following fixation onset $(0-20 \mathrm{~ms})$ in line with previous FRP studies (Hutzler et al., 2007; Dimigen et al., 2011 for review). This was done because of the possibility that some saccade-related activity may contaminate the EEG in the time immediately prior to fixation, making such a baseline correction relative to the pre-stimulus activity problematic. Trials containing EEG artefacts exceeding $+/-80 \mu \mathrm{V}$ were excluded from analysis. Averages were then formed for the upright and inverted faces and watch conditions at two time-points in the trials: The stimulus presentation ERP and the fixation FRP.

\section{Data analyses}

N170 responses have typically been observed in the occipito-temporal regions corresponding to the P7 and P8 electrodes in our study (see Rossion \& Jacques, 2007 for review). Visual inspection of the average waveforms to the peripheral presentation of upright faces (see Figure 2, top row) in Experiment 1 revealed that the mean N170 peak occurred at approximately $160 \mathrm{~ms}$ over occipito-temporal areas, corresponding to the left P7 and right P8 electrode sites, similar to previous ERP studies. The topographical scalp map of the difference between upright faces and watches at fixation suggested a difference localised to the occipital lobe, which was an unexpected finding. To explore face-sensitive differences in the typical occipito-temporal regions, as well as unexpected occipital region, we compared condition-specific brain potential differences in the left P7, right P8 occipito-temporal electrodes, and the central occipital Oz electrode.

Some conditions did not elicit distinct brain potential peaks, which render traditional peak-detection algorithms problematic. Thus, in line with previous research (Dimigen et al., 2011; Cauchoix et al., 2014), we used repeated-measures, two-tailed, permutation tests based on the t-max statistic (Blair \& Karniski, 1993) to determine if a statistically reliable facesensitive N170 (i.e., significantly larger to faces than watches) was elicited at both stimulus 
presentation and at first fixation, or at presentation only. An added benefit of this permutation approach is that it did not require us to predict the exact timing of the N170 elicited by selfguided fixations, which may well be different to the N170 elicited by an externally controlled presentation of a face.

The permutation tests were achieved through a series of sample-by-sample t-tests performed between the waveforms of the upright face and watch conditions in the first 250 ms following Presentation and Fixation separately. The t-max permutation procedure controls for the family-wise error rate in analyses involving multiple electrodes by randomly-shuffling (permuting) the data from all scalp electrodes and estimating the distribution of the nullhypothesis (100,000 permutations, in the current study). The largest observed t-values (t$\max$ ) for each comparison were then used to measure the time at which a significant difference between the experimental conditions manifested at the 0.05 and 0.01 alpha levels with 16 degrees of freedom. These comparisons of face-sensitivity were performed at the left P7, central Oz, and right P8 electrodes for Presentation and Fixation waveforms. Similar separate comparisons were conducted to compare the effects of face-inversion, where the difference between upright and inverted faces were also subjected to the t-max permutation procedure. Scalp-wide permutation analyses were additionally conducted and presented to provide insight and a visual depiction of face-sensitivity and orientation effects across all electrodes.

\section{Results}

\section{Face-sensitivity of the N170 at stimulus onset}

The waveforms elicited by the presentation of a stimulus in the periphery was significantly larger to upright faces than upright watches at P7 $(p<0.05$ from 125-171 ms, $p$ $<0.01$ from 137-166 ms), Oz ( $p<0.05$ from 134-223 ms, $p<0.01$ from 136-220 ms) and P8 ( $p<0.05$ from 178-194 ms, $p<0.01$ from $185-191 \mathrm{~ms}$ ). The times at which this face- 
sensitivity exceeded these alpha thresholds are depicted in Figure 2, and are in line with previous ERP studies involving the N170 (Rossion \& Jacques, 2007 for review).

A similar pattern was observed when the number of trials in the upright face condition was reduced (randomly selected) to match the upright watch condition, with significantly larger N170 responses to upright faces at P7 $(p<0.05$ from $129-171 \mathrm{~ms}, p<0.01$ from $145-149$ ms), Oz ( $p<0.05$ from 134-223 ms, $p<0.01$ from 139-219 ms) and P8 $(p<0.05$ from 179$194 \mathrm{~ms}, p<0.01$.

\section{Face-sensitivity of the N170 at first fixation}

Face-sensitivity was not reliably observed in the occipito-temporal P7 and P8 electrodes at stimulus fixation. However, early brain potentials were found to be significantly larger to watches than to faces at the central Oz electrode, arising at 82-92 ms $(p<0.05)$; see Figure 2), corresponding to the time-period between the P1 and N170 peaks. This pattern and time-window of significance was also obtained when the number of trials in the face condition matched that of the watch condition.

\section{Please insert Figure 2 about here}

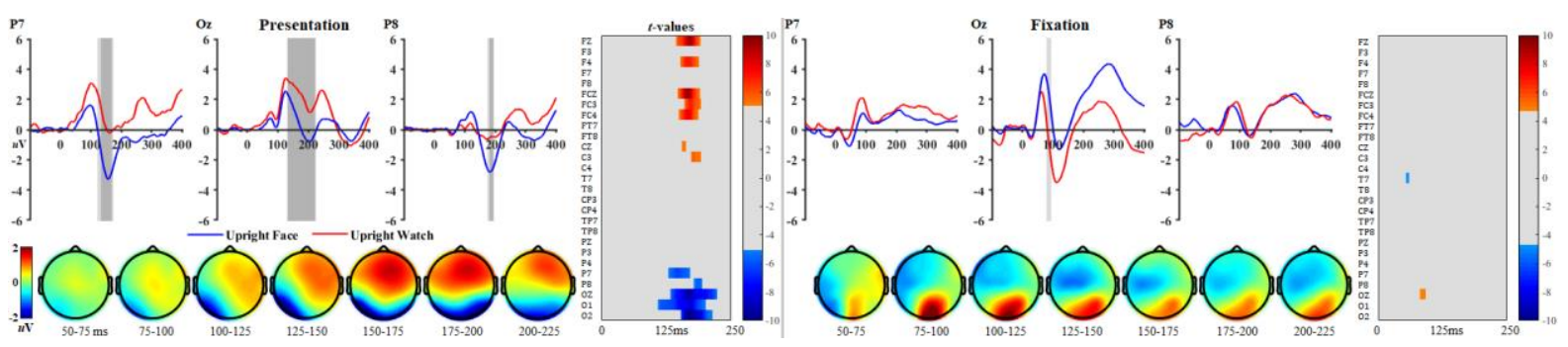

Figure 2. Face-sensitivity: Waveforms, topographic voltage maps, and scalp-wide t-value (thresholded at $p=0.05$ ) plots for the brain potential waveforms time-locked to the presentation of (left) and subsequent fixation on (right) face and watch stimuli using a right visual field presentation. Shaded periods in the waveforms denote the time-range of significance (light grey: $p<0.05$, dark grey: $p<0.01$ ). Topographic voltage maps represent the activity of faces minus watches for both Presentation and Fixation.

\section{Face-sensitivity of the N170 to inversion}

The comparison of upright and inverted faces at stimulus onset revealed a significantly greater negative activation in response to inverted faces than upright faces at the 
right occipito-temporal P8 electrode ( $p<0.05$ from 184-207), but not at the left occipitotemporal P7 or central occipital Oz electrodes (Figure 3). There were no reliable effects of face inversion observed at first fixation in either the occipito-temporal or central occipital regions.

Please insert figure 3 about here

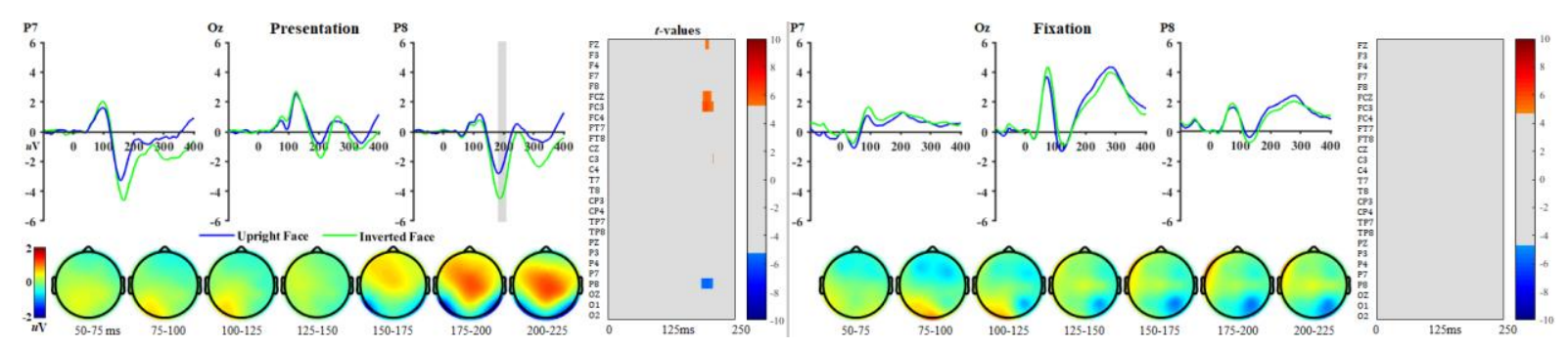

Figure 3. Face-inversion: Waveforms, topographic voltage maps, and scalp-wide t-value (thresholded at $p=0.05$ ) plots for the brain potential waveforms time-locked to the presentation of (left) and subsequent fixation on (right) upright and inverted faces using a right visual field presentation. Shaded periods in the waveforms denote the time-range of significance (light grey: $p<0.05$, dark grey: $p<0.01$ ). Topographic voltage maps represent the activity of inverted faces minus upright faces for both Presentation and Fixation.

\section{Experiment 2}

The results of Experiment 1 suggest that a peripheral preview eliminates the face-sensitivity of the N170 for the first fixation, and yields no further face-inversion effects after the initial presentation. To further test the validity of the conclusion that the peripheral preview is directly responsible for the lack of face-sensitivity in the follow-up fixation, Experiment 2 sought to replicate the original peripheral-preview effects on first fixation, and then to systematically control the peripheral preview available to participants to see whether abolishing the preview led to face-sensitivity at fixation instead of at the initial presentation. This involved presenting both structurally-intact and phase-scrambled upright faces and watches in the periphery, where the phase-scrambled images were replaced with their structurally-intact counterparts during the orienting saccade. To rule out biasing object or 
face processing by projecting the images to only the right or left occipital regions, Experiment 2 involved stimulus presentation in both left and right visual fields.

\section{Method}

\section{Ethics}

The Human Ethics Committee at Macquarie University approved the methods and procedures used in this study.

\section{Participants}

Eighteen participants were recruited for the experiment and were included in the analysis (13 females, 16 right-handed). They were aged between 19 and 43 years (mean age $=$ 21.2 years, $\mathrm{SD}=6$ ). All participants had normal or corrected-to-normal vision, and gave their informed consent before participating in the study. Participants were offered \$30AUD for their time.

\section{Stimuli}

There were eight experimental conditions in Experiment 2: Intact and scrambled faces (i.e., with and without a structural preview) in the left and right periphery, and watches in the same four conditions. 66 trials were presented in each condition for a total of 528 trials in the testing sessions. Experiment 2 used the upright faces and wrist-watches from Experiment 1 to represent intact faces and intact watches. Additional stimulus sets were created by phasescrambling the face and watch stimuli, utilising a Fourier phase randomisation procedure that replaced the phase spectrum of the images with random values (Jacques \& Rossion, 2004; Näasänen, 1999; Rossion \& Caharel, 2011; Rousselet, Pernet, et al., 2008). The phasescrambled images were thus matched to their structurally-intact counterparts in their lowlevel visual attributes. This provided an important methodological control for comparisons within stimulus category where images at fixation were exactly the same, the only difference being whether a structurally-intact or phase-scrambled preview had been visible. 


\section{Procedure}

The trial procedure was the same as in Experiment 1, with some improvements. To avoid biasing the patterns of neural results by projecting visual information to mostly only one visual field as in Experiment 1, Experiment 2 involved the presentation of peripheral images in the left and right peripheral regions (one side per trial). To accommodate the additional visual space utilised in the experiment, the stimuli were presented on a 24 " LCD computer monitor (120 Hz refresh rate). A fixation on a centrally-presented cross triggered the onset of the trials, as in Experiment 1. Either structurally-intact or phase-scrambled faces or watches were then presented in either the left or right periphery. The experiment utilised an invisible boundary that triggered the update of the screen in the middle of an orienting saccade, which replaced the phase-scrambled images with their structurally-intact counterparts (see Figure 4 for trial sequence). This procedure was confirmed to provide accurate stimulus-update latencies, with no trials requiring exclusion due to improper update times.

In contrast to Experiment 1 where participants were instructed to look at and scan the presented images (free-viewing), an effort was made in Experiment 2 to direct participants' initial fixations to the centre of the images. This was done through the use of an orthogonal task by instructing participants that a digit would replace the images approximately $750 \mathrm{~ms}$ after their initial fixation, and that they were required to judge whether the presented digit was greater or less than 5 (Dehaene, Dupoux, \& Mehler, 1990; Kinoshita \& Hunt, 2008). The presented digits were located directly underneath the centre of the images, thus directing the participants to make fixations as close to the centre of the images as possible. This was done to decrease the likelihood of stimulus-related fixation patterns producing low-level visual property differences in the resulting brain-potentials time-locked to the fixations. 
The eye-movement processing, EEG co-registration, and ERP/FRP processing steps for Experiment 2 were the same as for Experiment 1, and participants' eye-movement behaviours were similarly analysed in terms of fixation latency (time between stimulus presentation and first fixation onset) and the duration of the first fixation made to the stimuli.

a

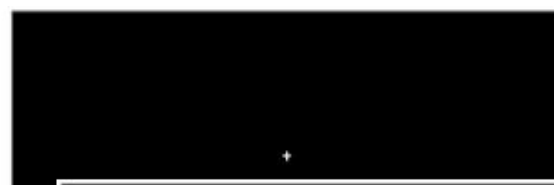

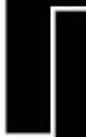
$150 \mathrm{~ms}$
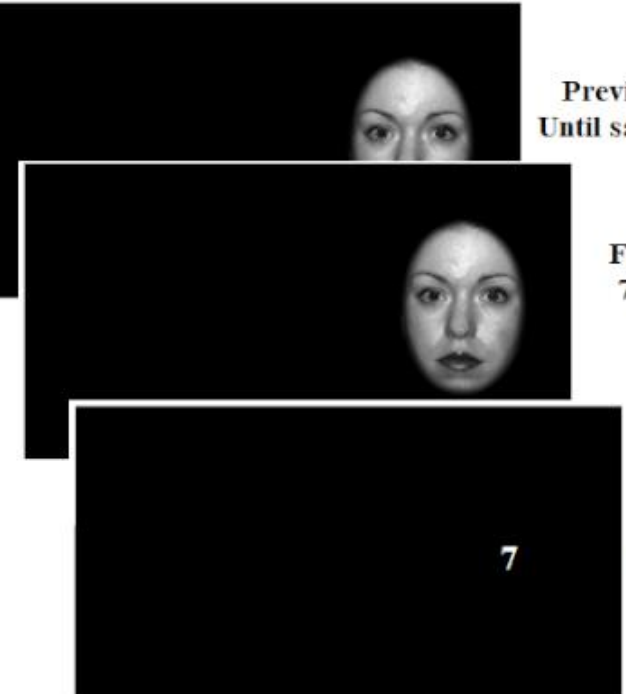

b Example scrambled stimuli
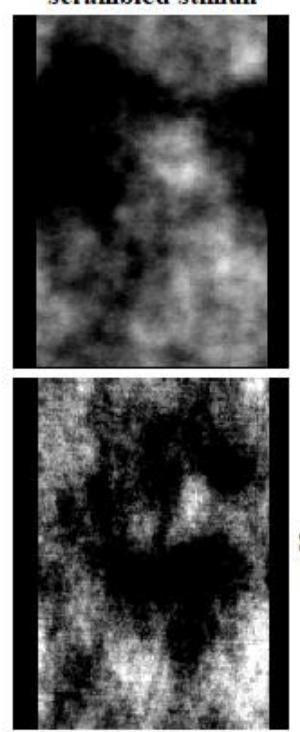

c
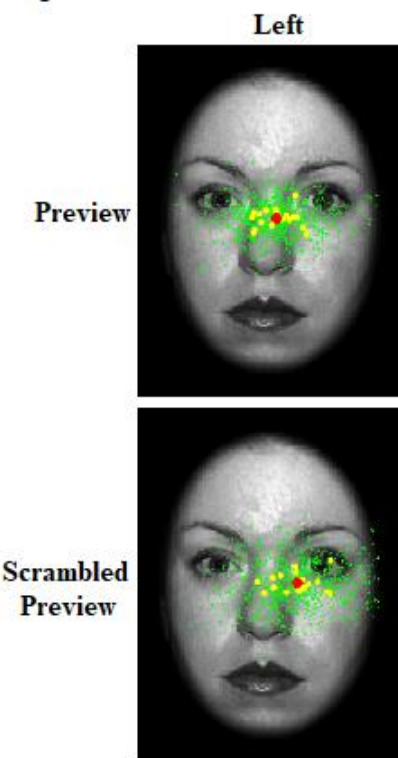
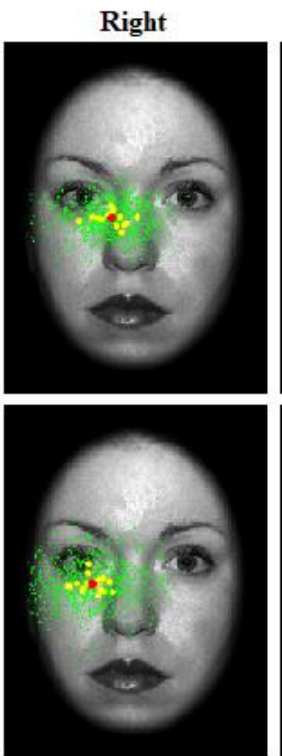
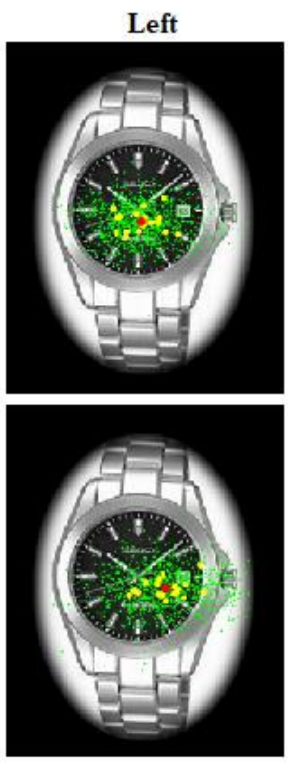

3
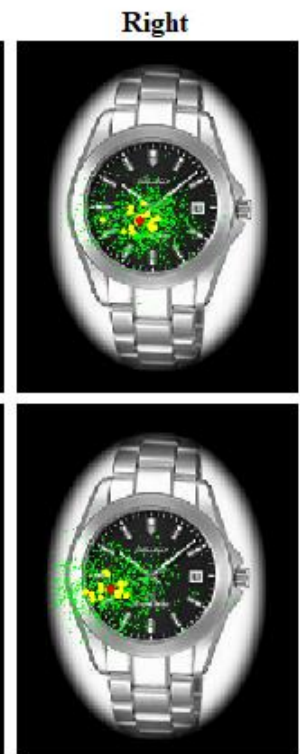

Please insert Figure 4 about here

Figure 4. Examples of the trial procedure (a), scrambled stimuli (b), and structurally-intact stimuli (c) used in experiment 2. The trial sequence involved a gaze-contingent update from a scrambled preview to a structurally-intact image during the saccade towards the stimuli, 
which was followed by a digit (orthogonal task). Coloured data points in figure 4c illustrate the first fixations made to the images presented in both left and right hemifields, in conditions with a structurally-intact peripheral preview (top) and with a phase-scrambled preview (bottom), representing the fixation locations of the group average (red), the subject average (yellow), and individual trials (green).

An ANOVA was conducted on fixation latency data from Experiment 2 comparing the effects of stimulus type (face/watch), preview type (intact/scrambled), and presentation hemifield (left/right). While there was no significant main effect of stimulus type $[F(1,17)=$ $2.41, p=.14]$, there was a significant main effect of presentation hemifield, with earlier fixations $(\sim 10 \mathrm{~ms})$ made to stimuli presented in the left hemifield $[F(1,17)=6.15, p=.024$, $\left.\eta^{2}=.27\right]$. There was also a significant main effect of preview type, with earlier fixations $(\sim 21$ ms) made when the preview was structurally-intact $\left[F(1,17)=19.53, p<.001, \eta^{2}=.53\right]$. An additional interaction between stimulus type and preview type was found $[F(1,17)=10.45, p$ $\left.=.005, \eta^{2}=.38\right]$, due to a larger effect of preview for face stimuli $(\sim 25 \mathrm{~ms})[F(1,17)=22.38$, $\left.p<.001, \eta^{2}=.57\right]$ than for watch stimuli $(\sim 18 \mathrm{~ms})\left[F(1,17)=15.23, p=.001, \eta^{2}=.47\right]$. It is likely that the faster saccadic responses to structurally-intact images reflected more efficient saccade planning, aided by the detection of the structures of the faces and watches.

An omnibus ANOVA conducted on the first fixation duration data revealed a significant main effect of preview, where intact previews led to longer fixations ( $73 \mathrm{~ms})$ than scrambled previews $\left[F(1,17)=29.4, p<.001, \eta^{2}=.63\right]$. No significant main effects were found for presentation hemifield $[F(1,17)=.71, p=.41]$ or for stimulus type $[F(1,17)$ $=2.28, p=.15]$. However, an interaction between preview type and stimulus type $[F(1,17)=$ $\left.11.28, p=.004, \eta^{2}=.39\right]$ was observed, due to fixation durations being approximately the same for faces and watches when the preview had been scrambled (297 ms and $296 \mathrm{ms,}$ respectively), while fixations to faces $(352 \mathrm{~ms})$ were significantly shorter than to watches $(388 \mathrm{~ms})$ when the preview had been intact $\left(\left[F(1,17)=4.67, p=.045, \eta^{2}=.22\right]\right.$. This latter result was not observed in Experiment 1, where initial fixations to faces and watches were of 
comparable duration. However, the task in Experiment 2 involved a judgement about a digit that was presented in the centre of where the face/watch image had been, $750 \mathrm{~ms}$ after initial fixation. It is therefore likely that the duration of the initial fixations to the images were influenced in part by this task, which served to direct attention to the centre of the face/watch stimuli.

Please insert Table 2 about here

\begin{tabular}{ccccccccc} 
& \multicolumn{4}{c}{ Structurally-Intact Preview } & \multicolumn{3}{c}{ Scrambled Preview } \\
\cline { 2 - 8 } & \multicolumn{2}{c}{ Faces } & \multicolumn{2}{c}{ Watches } & \multicolumn{2}{c}{ Faces } & \multicolumn{2}{c}{ Watches } \\
\cline { 2 - 8 } & Left & Right & Left & Right & Left & Right & Left & Right \\
\cline { 2 - 8 } $\begin{array}{c}\text { Trials - } \\
\text { Presentation }\end{array}$ & $62(2.4)$ & $61(3.3)$ & $63(3.4)$ & $61(3.9)$ & $61(4.3)$ & $60(4)$ & $62(3.6)$ & $59(4.9)$ \\
\hline $\begin{array}{c}\text { Trials - } \\
\text { Fixation }\end{array}$ & $63(2.6)$ & $62(3.6)$ & $63(3.3)$ & $61(4.1)$ & $62(4.3)$ & $60(4.1)$ & $63(3.7)$ & $60(5.1)$ \\
\hline $\begin{array}{c}\text { Fixation } \\
\text { latency (ms) }\end{array}$ & $264(27)$ & $274(32)$ & $264(26)$ & $276(32)$ & 292(46) & $300(54)$ & $283(44)$ & $298(50)$ \\
\hline $\begin{array}{c}\text { Fixation } \\
\text { duration (ms) }\end{array}$ & $345(143)$ & $359(124)$ & $373(126)$ & $403(139)$ & $293(106)$ & $301(97)$ & $297(106)$ & $295(80)$
\end{tabular}

Table 2. Mean trial numbers, fixation latencies (period between the stimulus presentation and subsequent fixation) and durations of first fixations on the images for all conditions in Experiment 2 in milliseconds (standard deviation in brackets).

\section{Results}

\section{Face-sensitivity of the N170 with structurally-intact preview}

As in Experiment 1, faces elicited larger N170 peaks than watches in response to both left peripheral presentations (P7: $p<0.05$ from 184-209 ms, $p<0.01$ from 188-207 ms; P8: $p$ $<0.05$ from 153-167 ms) and right peripheral presentations (P7: $p<0.05$ from 134-196 ms, $p$ $<0.01$ from 136-194 ms; Oz ( $p<0.05$ from 192-196 ms).

At first fixation, no face-sensitivity was observed in the occipito-temporal region (i.e., P7 and P8 electrode sites). However, for both left and right peripheral presentations, watches elicited significantly greater negative peaks at the central occipital Oz electrode $(p<0.05$ from 113-179 ms, $p<0.01$ from 116-177 ms and $p<0.05$ from $115-163 \mathrm{~ms}, p<0.01$ from 
118-162, respectively, see Figure 5). This was a similar pattern to that observed in Experiment 1 at the central occipital electrode, although the effect occurred approximately $100 \mathrm{~ms}$ later in Experiment 2.

Please insert Figure 5 about here
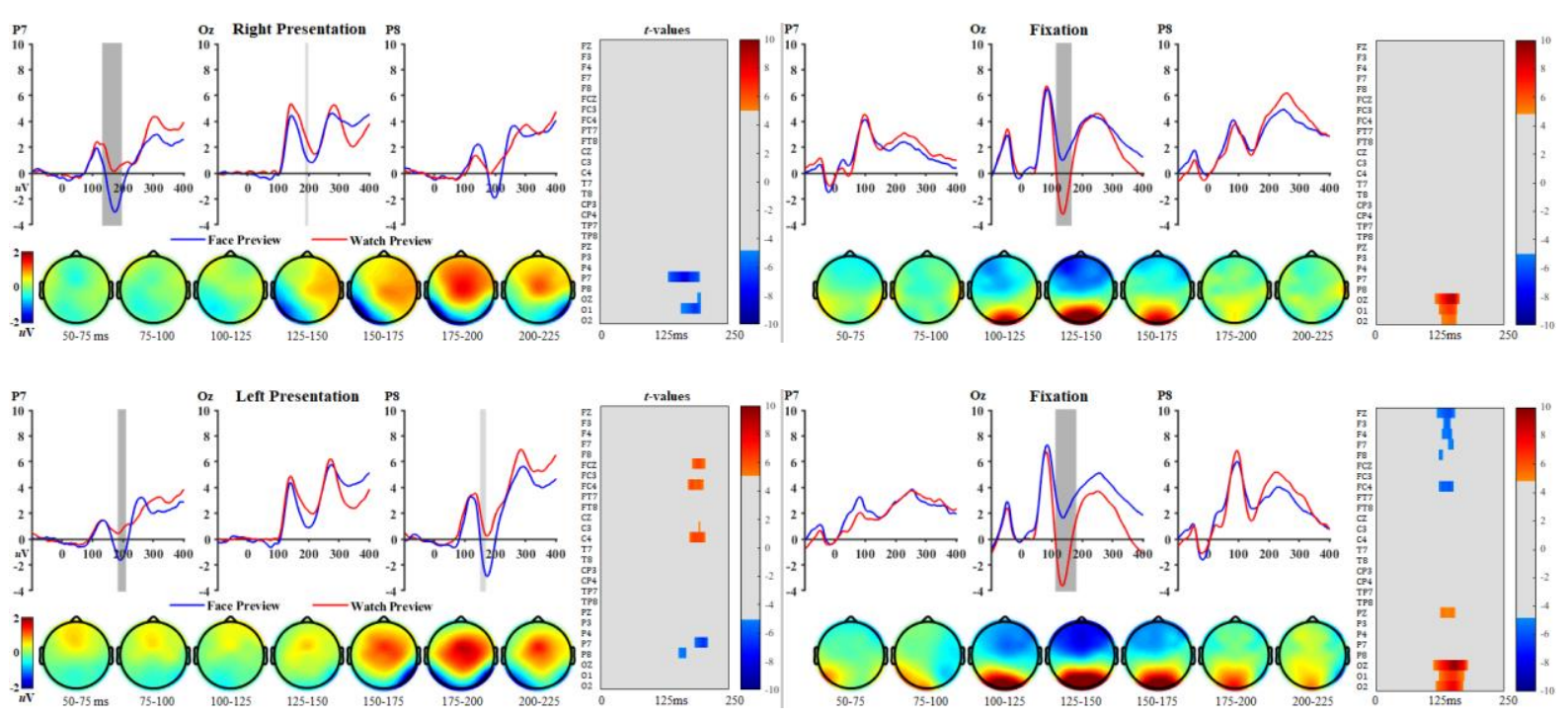

Figure 5. Face-sensitivity - Structurally-intact peripheral preview: Waveforms, topographic voltage maps, and scalp-wide $t$-value (thresholded at $p=0.05$ ) plots for the brain potential waveforms time-locked to the presentation of (left) and subsequent fixation on (right) face and watch stimuli for both right (top) and left (bottom) visual field presentations. Shaded periods in the waveforms denote the time-range of significance (light grey: $p<0.05$, dark grey: $p<0.01$ ). Topographic voltage maps represent the activity of faces minus watches for both Presentation and Fixation.

\section{Face-sensitivity of the N170 with phase-scrambled preview}

There were no significant differences between the mean N170 for phase-scrambled faces and watches at stimulus onset. However, at first fixation (when the images had been updated to their structurally-intact counterparts) faces elicited significantly larger negative brain potentials during the N170 time period than watches, though the pattern of significance changed depending on the visual field of stimulus presentation (Figure 6). For left visual field presentations, faces elicited larger negative amplitudes at the right P8 occipito-temporal electrode ( $p<0.05$ from 132-163 ms, $p<0.01$ from 138-159 ms), and for right visual field, this effect was observed in the left P7 electrode ( $p<0.05$ from 149-169 ms). At the central occipital Oz site, watches elicited a larger negative brain potential than faces for left visual 
field presentations only ( $p<0.05$ from 103-163 ms, $p<0.01$ from 106-159 ms), as well as a later period of significance arising at approximately $200 \mathrm{~ms}(p<0.05$ from 206-228 ms, $p<$ 0.01 from $217-222 \mathrm{~ms})$.

Please insert Figure 6 about here
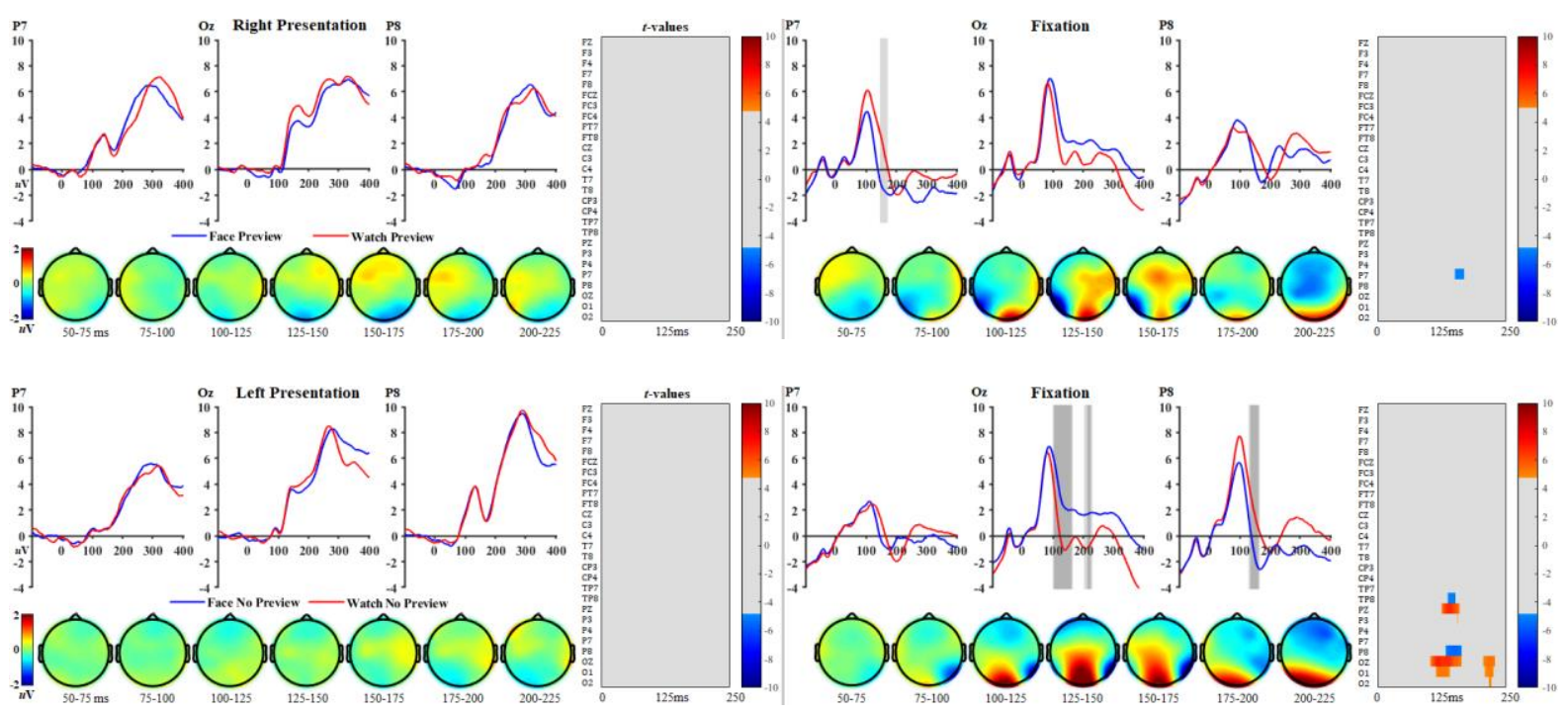

Figure 6. Face-sensitivity - phase-scrambled peripheral preview: Waveforms, topographic voltage maps, and scalp-wide $t$-value (thresholded at $p=0.05$ ) plots for the brain potential waveforms time-locked to the presentation of (left) and subsequent fixation on (right) face and watch stimuli for both right (top) and left (bottom) visual field presentations. The stimuli in the peripheral-preview were phase-scrambled, and were updated to their structurallyintact counterparts during the orienting saccade before fixation. Shaded periods in the waveforms denote the time-range of significance (light grey: $p<0.05$, dark grey: $p<0.01$ ). Topographic voltage maps represent the activity of faces minus watches for both Presentation and Fixation.

\section{Peripheral preview effect for faces - Scrambled versus intact preview}

Structurally-intact peripheral previews of the face stimuli at stimulus onset elicited significantly larger brain potentials in the occipito-temporal and central occipital regions than their phase-scrambled counterparts. Both right visual field presentations ( $\mathrm{P} 7: p<0.05$ from 132-250 ms, $p<0.01$ from 134-250 ms; P8: $p<0.05$ from 193-222, $p<0.01$ from 196-219 ms); Oz: $p<0.05$ from 205-250 ms, $p<0.01$ from 210-250 ms), and left visual field presentations (P: $p<0.05$ from 177-236 ms, $p<0.01$ from 179-234 ms; P8: $p<0.05$ from 151-250, $p<0.01$ from 156-250 ms; Oz: $p<0.05$ from 189-250 ms, $p<0.01$ from 192-250 ms) elicited more negative potentials during the N170 time range. An earlier effect 
corresponding to the $\mathrm{P} 1$ peak was also observed at $\mathrm{P} 8$ for a right visual field presentation $(p<$ 0.05 from $122-157 \mathrm{~ms}, p<0.01$ from $124-155 \mathrm{~ms}$ ).

In contrast to the N170 responses to peripheral preview at stimulus onset, the N170 responses at first fixation were significantly more negative for phase-scrambled faces than structurally-intact faces (Figure 7). This effect was limited to the occipito-temporal regions for presentations in both the right (P7: $p<0.05$ from 225-250 ms, $p<0.01$ from 235-250 ms; P8: $p<0.05$ from 170-193) and left visual fields (P7: $p<0.05$ from 174-204 ms, $p<0.01$ from 177-201ms; P8: $p<0.05$ from 161-250, $p<0.01$ from 176-250). There were additional transient periods of significance $(p<0.05)$ at the P7 electrode for left visual presentations at 209-211 ms and 240-250 ms.

Please insert Figure 7 about here
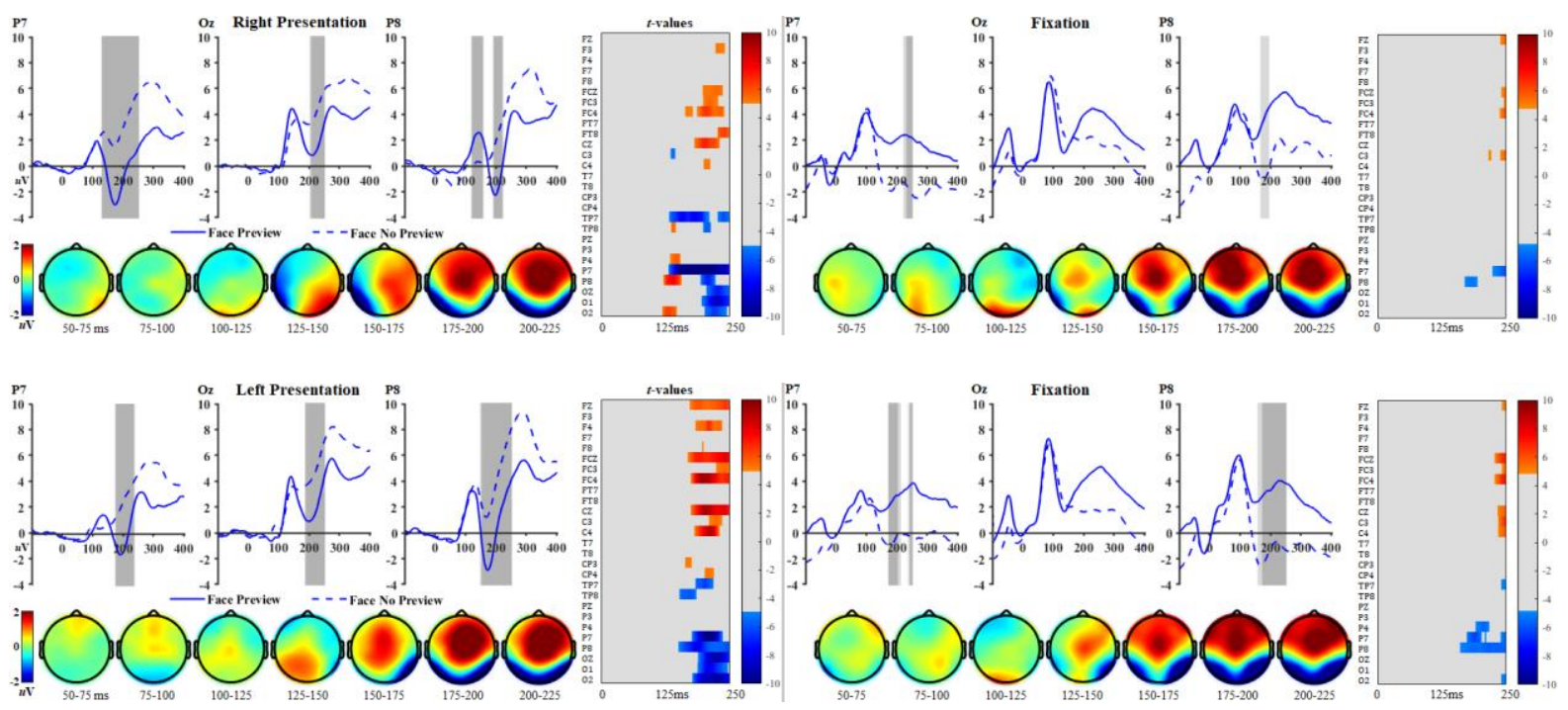

Figure 7. Preview-sensitivity - Faces: Waveforms, topographic voltage maps, and scalpwide $t$-value (thresholded at $p=0.05$ ) plots for the brain potential waveforms time-locked to the presentation of (left) and subsequent fixation on (right) face stimuli for both right (top) and left (bottom) visual field presentations. The stimuli in the peripheral-preview were phasescrambled, and were updated to their structurally-intact counterparts during the orienting saccade before fixation. Shaded periods in the waveforms denote the time-range of significance (light grey: $p<0.05$, dark grey: $p<0.01$ ). Topographic voltage maps at Presentation represent the activity of structurally-intact faces minus scrambled faces, and at Fixation they represent activity of scrambled faces minus intact faces. 
The effect of a structurally-intact peripheral preview of watch stimuli at stimulus onset was similar to faces, with larger negative activity elicited in the N170 time range when a preview was available at occipito-temporal and central occipital regions for both right presentation (P7: $p<0.05$ from 244-245 ms; P8: $p<0.05$ from 235-250 ms; Oz: $p<0.05$ from 211-250 ms, $p<0.01$ from 213-250 ms), and left presentation (P7: $p<0.05$ from 197$250 \mathrm{~ms}, p<0.01$ from 207-250 ms; P8: $p<0.05$ from 222-250, $p<0.01$ from 225-250 ms; Oz: $p<0.05$ from 207-250 ms, $p<0.01$ from 211-250 ms).

At first fixation, these patterns once again reversed, with larger negative brain potentials elicited when the peripheral preview was of phase-scrambled watch stimuli for both right presentation (P7: $p<0.05$ from 196-250 ms, $p<0.01$ from 207-250 ms; P8: $p<$ 0.05 from 174-250 ms, $p<0.01$ from 177-250 ms; Oz: $p<0.05$ from 199-239 ms), and left presentation (P7: $p<0.05$ from 183-250 ms, $p<0.01$ from 193-250 ms; P8: $p<0.05$ from 183-248, $p<0.01$ from 191-235 ms; Oz: $p<0.05$ from 223-250 ms.

While scrambled watches elicited significantly larger negative peaks at the occipitotemporal P7/P8 electrodes, the reverse was found at the central occipital Oz electrode (Figure 8), where structurally-intact faces elicited significantly larger early negative peaks $(p<0.05$ from 109-141 ms, $p<0.01$ from 110-138 ms).

Please insert Figure 8 about here

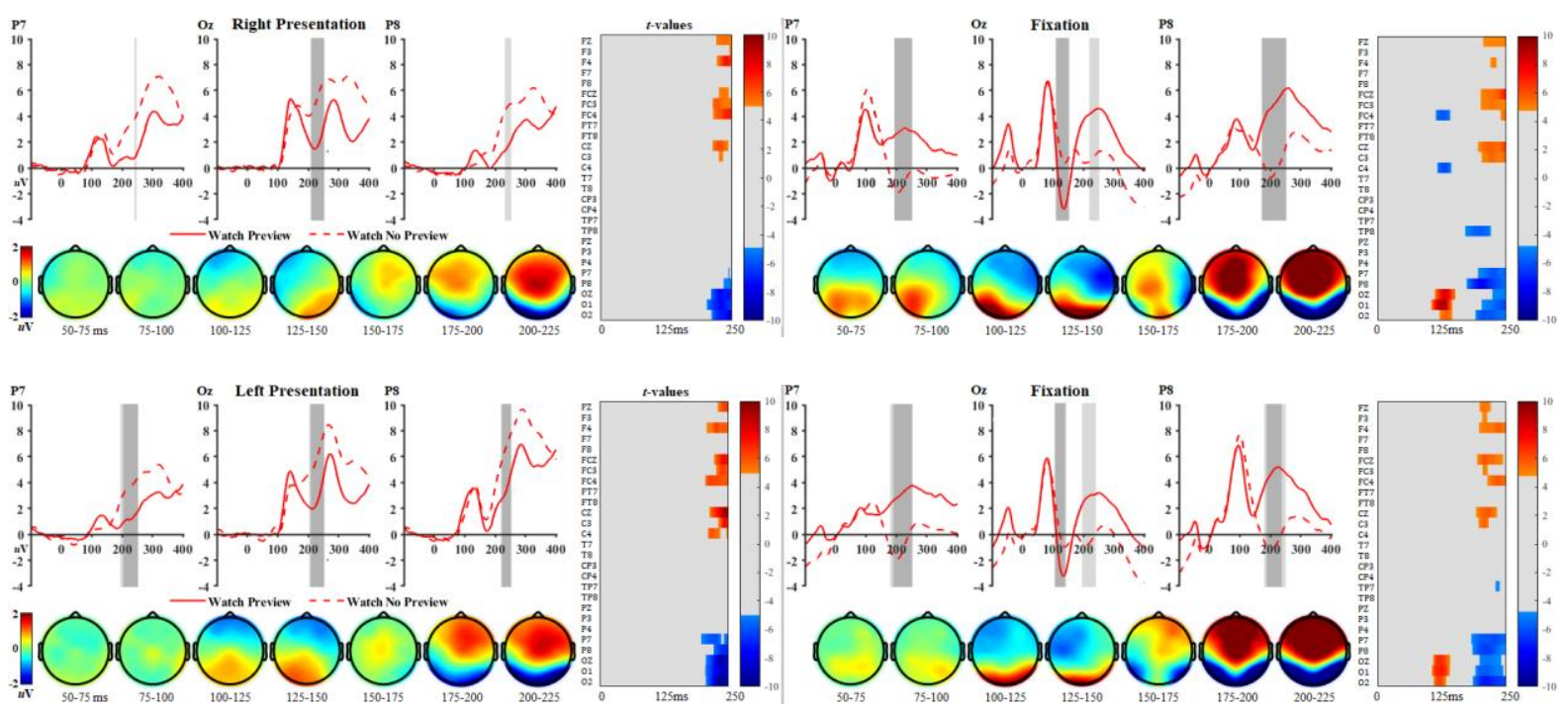


Figure 8. Preview-sensitivity - Watches: Waveforms, topographic voltage maps, and scalpwide $t$-value (thresholded at $p=0.05$ ) plots for the brain potential waveforms time-locked to the presentation of (left) and subsequent fixation on (right) watch stimuli for both right (top) and left (bottom) visual field presentations. The stimuli in the peripheral-preview were phasescrambled, and were updated to their structurally-intact counterparts during the orienting saccade before fixation. Shaded periods in the waveforms denote the time-range of significance (light grey: $p<0.05$, dark grey: $p<0.01$ ). Topographic voltage maps at Presentation represent the activity of structurally-intact watches minus scrambled faces, and at Fixation they represent activity of scrambled watches minus intact faces.

\section{Discussion}

\section{Experiment 1}

Experiment 1 sought to determine whether face-sensitivity is observed in response to both peripheral preview and subsequent fixation by comparing the N170 elicited by faces and objects, and whether the effects of face inversion modulate the N170 beyond the initial presentation. The onset of face stimulus in the visual periphery elicited a distinct facesensitivity effect over occipito-temporal and central occipital electrodes, which was comparable to patterns observed in similar paradigms involving peripheral presentation of faces (Rousselet et al., 2005). In addition, we observed a face inversion effect over the right occipito-temporal electrode, comparable to previous studies showing an amplitude enhancement observed in response to face inversion (Kovács et al., 2017). Critically, no significant amplitude differences were observed at either the left or right occipito-temporal (P7/P8) electrodes at first fixation to the stimulus. There was, however, a period of significant difference between the conditions at the Oz electrode midway between the P1 and N170 peaks, with the watch stimuli eliciting a significantly more negative response than faces.

At first fixation, occipito-temporal brain potentials during the N170 time window were attenuated regardless of condition. An exception to this was observed at the centraloccipital region, measured at the $\mathrm{Oz}$ electrode, where the watch stimuli elicited a significantly more negative amplitude midway between the P1 and N170 peaks (Figure 2). While the effects in this experiment were predicted to occur at occipito-temporal regions (i.e., our P7 
and P8 electrode sites), it is possible that a peripheral preview may lead to attentional modulation that manifests in brain potentials arising earlier than the N170, similar to effects observed for parafoveal preview benefits in word reading (Kornrumpf et al., 2016; Niefind \& Dimigen, 2016). Given that faces have typically been found to attract greater levels of attention than other stimulus categories (Palermo \& Rhodes, 2007), it is possible that attention-modulated early occipital P1 activity due to attentional capture from faces may be in part responsible for this effect. The timing of the effect, however, suggests that a combination of different processes may be responsible for this difference, as the effect falls between the classically observed P1 and N170 peaks.

It is noteworthy that we failed to observe the face-inversion effect at first fixation. As the N170 has been found to be a sensitive index of the holistic processing of faces (Itier \& Taylor, 2004; Itier et al., 2006; Linkenkaer-Hansen et al. 1998; Rossion et al., 2000), these results suggest that the processes that give rise to the increased N170 potentials at stimulus onset are completed before the advent of the first fixation.

\section{Experiment 2}

Experiment 2 sought to systematically test the contribution of the peripheral preview to the lack of face-sensitivity at fixation by phase-scrambling the preview of the faces and watches in half of the trials, and updating them to their structurally-intact counterparts midsaccade. Replicating the results of Experiment 1, the results of Experiment 2 reinforced the finding that structurally-intact faces elicited a significantly larger N170 potential in the periphery at stimulus onset, but not in a subsequent fixation. However, when this peripheral preview was phase-scrambled, faces elicited significantly larger N170 responses at first fixation than watches. This suggests that the neural responses at first fixation are analogous, in principle, to those observed in traditional ERP studies, and that a structural preview of 
images will significantly modulate and attenuate neural patterns when they are centrally fixated.

Experiment 2 further highlighted the role of peripheral preview for the processing of both face and watch stimuli, with larger negative brain potentials observed at first fixation when this preview was prevented by phase-scrambling the image. This finding suggests that the preview effects are not face-specific, which aligns with findings of previous traditional ERP studies which report the N170 to be face-sensitive but not face-specific, and to be elicited by a range of stimulus categories of varying expertise (Rossion, Kung, \& Tarr, 2004; Tanaka \& Curran, 2001; Ip, Wang, \& Fu, 2017).

Experiment 2 further clarified that face-sensitivity was significantly modulated by the peripheral preview in both right and left peripheral stimulus presentations. The initial N170 responses to peripheral presentation was related to the hemifield of presentation, with larger amplitudes observed in contralateral hemispheres, an effect similar to that observed by Rousselet et al. (2005), where spatially-scaled faces elicited larger N170 responses in the hemispheres contralateral to presentation. While it might have been predicted from previous findings that faces would exhibit larger N170 responses in the right hemisphere in general (Dundas, Plaut, \& Behrmann, 2015; Rossion et al., 2003; Scott \& Nelson, 2006), we did not observe such a pattern. On the contrary, face-sensitivity manifested earlier in the contralateral hemisphere when stimuli were presented in the right hemifield than in the left in Experiment 2 (figure 5). While the current study is limited in its ability to shed light on the source of this unexpected pattern, the FRP paradigm would be well-suited to investigate questions of the lateralisation of processing for faces and other visual categories. This is especially true of visual processes where peripheral or parafoveal previews are part of dynamic perception and integration of stimuli across time, such as in word reading (Dundas, Plaut, \& Behrmann, 2015). 
In order to properly address the question of hemispheric preference or lateralisation for processing different kinds of visual stimuli in both presentations and fixations it is necessary to control the landing positions of fixations made to such stimuli. The reason for this is that while fixations may generally land in the centre of a stimulus, small differences in landing position will lead to visual information being projected to slightly different places in the visual field. If there are systematic differences in landing position between two conditions or stimulus categories, these low-level properties may lead to differences in the resulting brain-potentials time-locked to fixations. While face-sensitivity has been found to persist when fixations are made to various areas within a face and object stimulus (de Lissa et al., 2014; Nemrodov et al., 2014), effects that are less robust might not be reliably measured without controlling for such low-level differences. Accordingly, care is necessary in the design of such paradigms to balance or control these factors either through stimulus presentation control or through post-hoc analyses, where nuanced effects may otherwise be influenced by small differences in low-level properties.

An unexpected finding from both Experiments 1 and 2 was a difference between the watch and face stimuli at first fixation over the central occipital electrode. The timing of this difference in Experiment 1 fell between the P1 and N170 peaks, suggesting a possible contribution of both brain potentials to the effect. However, the occipital difference in Experiment 2 was consistently observed in the N170 latency range, and consistently larger for fixations on the watch stimuli than fixations on the faces when peripheral preview of the stimuli was available. The relevance of the preview was further highlighted by the striking disparity that was observed at first fixation between the occipito-temporal and occipital N170 responses when a peripheral preview had been available compared to when it had not (Figures 7 and 8). When a structurally-intact peripheral preview was available, the occipitotemporal N170 was significantly smaller than when the preview had been phase-scrambled, 
while the occipital N170 was significantly larger when a preview had been available compared to when it had not been. Notably, this occipital N170 effect was not found for the face stimuli, where the presence or absence of peripheral preview did not appear to modulate this central occipital brain potential (Figure 7).

These results seem to suggest a dissociation of mechanisms, with the occipitotemporal activations reflecting a categorical detection/encoding process and the central occipital activation reflecting an object-related form of analysis that benefits from a structural preview. Topographical differences in N170 (or N1) neural responses to faces and objects have been observed in previous ERP studies, suggesting differences in laterality as well as differences in the spatial locations of the neural populations recruited by stimuli belonging to various categories (Itier \& Taylor, 2004; Rossion et al., 2003). These findings are in line with fMRI results suggesting both an overlap and a dissociation of neural areas recruited during the processing of objects and faces (Gao et al., 2018; Kanwisher \& Yovel, 2006). However, differences in neural activity elicited by faces and objects are not typically observed in the occipital area when time-locking to discrete visual presentations, as noted by Gao et al. (2018). The occipital N170 sensitivity to watches observed in Experiment 2, especially when a peripheral preview was available, may therefore be due to the nature of the paradigm employed in this study - visual presentations that are under participants' control through eyefixations may involve peripheral previews that modulate not only the strength of neural responses, but where such effects might manifest.

Experiment 2 also provides a useful methodological insight into the effects of using a short $20 \mathrm{~ms}$ post-fixation period for baseline-correction of fixation-related potentials rather than a more typical pre-stimulus presentation baseline. Brain potentials time-locked to fixations carry the possibility that activity preceding the fixation might be modulated by condition. This was observed in both experiments 1 and 2, where fixations on the faces and 
watches were preceded by face-sensitive differences in response to peripheral presentation. The use of a short post-fixation period as a baseline thus served to avoid using a baseline potentially contaminated with condition-specific effects. The reliability of using such a baseline was attested by the results of the comparisons in Experiment 2 involving phasescrambled images and their structurally-intact counterparts as peripheral previews, such that when the images were subsequently fixated they were exactly the same. The overlap of the early parts of the waveforms (especially the P1 peaks) in these comparisons (figures 7 and 8) attests to the reliability of this baseline, suggesting it to be a useful point of measurement in some FRP paradigms where taking a pre-stimulus baseline may not be appropriate.

\section{General Discussion}

The overall aim of the study was to investigate dynamic face processing by coregistering EEG and eye-movement recordings. The results of Experiment 1 revealed that the face-sensitive N170 peaks observed in response to a stimulus presented in the periphery is not elicited in a subsequent fixation to that stimulus. Experiment 2 clarified that when the peripheral preview is prevented by phase-scrambling the images, the face-sensitivity of the N1 is observed at first fixation on the stimulus. The findings support the hypothesis that the N170 in part reflects a detection process (Bentin et al., 1996; George et al., 2005), and that once this process is triggered, it is not recruited in a follow-up fixation. This interpretation is further supported and extended by the comparison of preview effect for faces and watches separately, which showed that the N170 at Fixation was significantly modulated by a peripheral preview for both stimulus categories. Such a finding is also in line with the objectsensitivity observed in N170 responses in previous studies (Rossion, Kung, \& Tarr, 2004; Tanaka \& Curran, 2001; Ip, Wang, \& Fu, 2017). The effect of a peripheral preview may therefore be seen as involving a stimulus-category detection process that is activated in response to a stimulus presentation. When a structurally-intact preview is prevented at 
presentation, as in Experiment 2, this category-detection process is not able to operate, and is therefore triggered when a fixation is made on the structurally-intact image for the first time, allowing for the structural analysis necessary for categorical detection to occur.

An alternate interpretation of the absence of a face-sensitive N170 at first fixation on a peripheral stimulus is the possible mechanism of habituation or repetition suppression. Some studies have found that when an individual face is presented twice in succession, the N170 response to the repeat presentation is significantly attenuated (Itier and Taylor, 2002, 2004; Jacques, d'Arripe, \& Rossion, 2007; Kovács et al., 2006, 2007; Turano et al., 2017; Vizioli et al., 2010; Heisz et al., 2006). The repetition-suppression paradigm conceptually overlaps with one involving a peripheral presentation followed by a subsequent fixation. In both paradigms, there is a temporal gap between the two visual events entering the retina that may entail a process of habituation or adaptation in which the neural responses are decreased by having previously responded to the same visual stimulus (Schweinberger \& Neumann, 2016 for review of repetition suppression). However, the reduction in N170 response to face repetition has not been observed consistently, with some repetition suppression studies not finding N170 attenuation (Huddy et al., 2003; Jemel et al., 2003; Mnatsakanian \& Tarkka, 2004; Schweinberger et al., 1995, 2002, 2004). Moreover, these paradigms typically involve initial (adapter) face presentations of $2+$ seconds, which is much longer than the peripheralpreviews were available in the current experiments. In the current studies participants were encouraged to make eye-movements to the images when they appeared, and fixation-onset latencies from the eye-movement data suggested that the time between peripheral preview and stimulus fixation was on average less than $300 \mathrm{~ms}$ (Tables 1 and 2). While the results of Experiment 1 and 2 suggest that a peripheral preview of a face leads to detection and structural encoding that is not exhibited in a subsequent fixation, it is yet unknown how long this effect lasts. It is possible that face representations that are encoded when a face is 
presented may degrade over time, or that additional factors such as shifts in visual attention may cause a resetting of this process, where a face-sensitive N170 would be further elicited at fixation.

It therefore remains to be determined whether the significant attenuation of brain potentials at fixation caused by peripheral preview in the current study relates to the same mechanisms of habituation or adaptation found in repetition-suppression paradigms, or a categorically different mechanism.

The current results may generalise to FRP paradigms involving a peripheral (or indeed parafoveal) preview, where N170 effects elicited by a preview may not be observable at fixation but rather earlier, such as in free-viewing paradigms. This raises a complexity for paradigms seeking to investigate face and object processing in more ecologically-valid contexts that allow natural fixations to be made - systematically controlling the stimuli may intrinsically require limiting the natural experience of participants.

While the current results indicate that a structural preview of a peripheral image significantly attenuates the N170 response at first fixation, the relevance of task has not yet been clarified with respect to this effect, as neither experiment 1 or 2 involved an explicit judgement of the stimuli. Apart from the potential for task and a corresponding modulation of visual attention to influence neural responses during face processing (Crist et al., 2008), freeviewing paradigms may entail a type of pre-processing of stimuli in peripheral or parafoveal regions that facilitates other kinds of processing at fixation.

The results of our study highlight the importance of preview and pre-processing on the face-sensitivity of N170 potentials elicited by subsequent fixations made on images. Conceptually, this result has broader implications to the study of brain potentials during dynamic visual processing, where natural fixations involving peripheral previews may not capture the full array of processing that is elicited in the analysis of objects. Consideration 
should therefore be given to when stimuli become available for visual analysis in such contexts, as this will allow a richer view of when and how the brain processes a complex visual environment. Accordingly, researchers utilising the flexibility of the FRP technique in such paradigms will benefit from systematically controlling, monitoring, and understanding the effect of peripheral previews when interpreting fixation-related brain activity. 


\section{Acknowledgements}

This work was supported with funding from a Macquarie University MQRES postgraduate grant, the Macquarie University Strategic Infrastructure Scheme, and the Experimental Psychology Society Study Visit Grant. Contributions- Original idea: RP, FH, SH, PdL, YM. Protocol development: RP, PdL, YM, FH, SH, GM. Data collection: PdL, YM, FD. Data processing and analyses: PdL. Writing of manuscript: PdL, GM, RP, FH, SH, YM, FD. 


\section{References}

Baccino, T., \& Manunta, Y. (2005). Eye-fixation-related potentials: insight into parafoveal processing. Journal of Psychophysiology, 19(3), 204-215.

Bentin, S., Allison, T., Puce, A., Perez, e., \& McCarthy, G. (1996). Electrophysiological studies of face perception in humans. Journal of Cognitive Neuroscience, 8(6), 551565.

Bentin, S., Degutis, J., D’Esposito, M., \& Robertson, L. (2007). Too many trees to see the forest: performance, event-related potential, and functional magnetic resonance imaging manifestations of integrative congenital prosopagnosia. Journal of Cognitive Neuroscience, 19(1), 132-146.

Bentin, S., \& Deouell, L.Y. (2000). Structural encoding and identification in face processing: ERP evidence for separate mechanisms. Cogn. Neuropsychol. 17, 35-54,

Blair, R.C., \& Karniski, W. (1993). An alternative method for significance testing of waveform difference potentials. Psychophysiology 30, 518-524.

Bruce, V., \& Young, A. (1986). Understanding face recognition. British Journal of Psychology, 77, 305-327.

Cauchoix, M., Barragan-Jason, G., Serre, T., \& Barbeau, E.J. (2014) the neural dynamics of face detection in the wild revealed by MVPA. Journal of Neuroscience 34, 846-854.

Crist, R.E., Wu, C.T., Karp, C., \& Woldorff, M. G. (2008). Face Processing is Gated by Visual Spatial Attention. Frontiers in human neuroscience, 1, 10.

Crouzet, S. M., Kirchner, H., \& Thorpe, S. J. (2010). Fast saccades toward faces: Face detection in just 100 ms. Journal of Vision, 10(4), 1-17. 16.

Degno, F., Loberg, O., Zang, C., Zhang, M., Donnelly, N., \& Liversedge, S. P. (2019) Parafoveal Previews and Lexical Frequency in Natural Reading: Evidence from Eye 
Movements and Fixation-Related Potentials. Journal of Experimental Psychology: General, 148(3), 453-474.

Dehaene, S., Dupoux, E., \& Mehler, J. (1990). Is numerical comparison digital: Analogical and Symbolic effects in two-digit number comparison. Journal of Experimental Psychology: Human Perception and Performance, 16, 626-641.

de Lissa, P., McArthur, G., Hawelka, S., Palermo, R., Mahajan, Y., \& Hutzler, F. (2014). Fixation location on upright and inverted faces modulates the N170. Neuropsychologia, 57, 1-11.

Delorme, A, \& Makeig, S. (2004) EEGLAB: an open source toolbox for analysis of singletrial EEG dynamics including independent component analysis. J. Neuroscience Methods, 134, 9-21.

Devillez, H., Guyader, N., Guérin-Dugué, A. (2015). An eye fixation-related potentials analysis of the P300 potential for fixations onto a target object when exploring natural scenes. Journal of Vision, 15 (13), 20.

Dimigen, O., Sommer, W., Hohlfeld, A., Jacobs, A., \& Kliegl, R. (2011). Coregistration of eye movements and EEG in natural reading: Analyses \& review. Journal of Experimental Psychology: General, 140(4), 552-572.

Dimigen, O., Kliegl, R., \& Sommer, W. (2012). Trans-saccadic parafoveal preview benefits in fluent reading: A study with fixation-related brain potentials. Neuroimage. 62, 381393.

Eimer, M. (1998). Does the face-specific N170 component reflect the activity of a specialized eye processor? Neuroreport, 9(13), 2945-2948.

Eimer, M. (2000). The face-specific N170 component reflects late stages in the structural encoding of faces. NeuroReport: For Rapid Communication of Neuroscience Research, 11(10), 2319-2324. 
Ekman, P., \& Friesen, W. V. (1976). Pictures of facial affect. Palo Alto, CA: Consulting Psychologists Press.

Gao, C., Conte, S., Richards, J.E., Xie, W., \& Hanayik, T. (2019). The neural sources of N170: Understanding timing of activation in face-selective areas. Psychophysiology, 56(6):e13336.

George, N., Jemel, B., Fiori, N., Chaby, L., \& Renault, B. (2005). Electrophysiological correlates of facial decision: insights from upright and upside-down Mooney-face perception. Cognitive Brain Research, 24, 663-673.

Graupner, S., Velichkovsky, B.M., Pannasch, S., \& Marx, J. (2007). Surprise, surprise: two distinct components in the visually evoked distractor effect. Psychophysiology, 44, $251-261$.

Guérin-Dugué, A., Roy, R. N., Kristensen, E., Rivet, B., Vercueil, L., \& Tcherkassof, A. (2018). Temporal Dynamics of Natural Static Emotional Facial Expressions Decoding: A Study Using Event- and Eye Fixation-Related Potentials. Frontiers in psychology, 9, 1190.

Gur, R.C., Sara, R., Hagendoorn, M., Marom, O., Hughett, P., Macy, L., \& Gur, R. E. (2002). A method for obtaining 3-dimensional facial expressions and its standardization for use in neurocognitive studies. Journal of Neuroscience Methods, 115, 137-143.

Guyader, N., Chauvin, A., Boucart, M., \& Peyrin, C. (2017). Do low spatial frequencies explain the extremely fast saccades towards human faces? Vision Research, 133, 100111.

Heisz, J. J., Watter, S., \& Shedden, J. M. (2006). Automatic face identity encoding atthe N170. Vision Research, 46(28), 4604-4614. 
Huber-Huber, C., Ditye, T., Fernández, M.M., \& Ansorge, U. (2016). Using temporally aligned event-related potentials for the investigation of attention shifts prior to and during saccades. Neuropsychologia, 92, 129-141.

Huddy, V., Schweinberger, S.R., Jentzsch, I., \& Burton, A.M. (2003). Matching faces for semantic information and names: An event-related brain potentials study. Cognitive Brain Research, 17, 314-326.

Hutzler, F., Braun, M., Vö, M. L. H., Engl, V., Hofmann, M., Dambacher, M. et al. (2007). Welcome to the real world: Validating fixation-related brain potentials for ecologically valid settings. Brain Research, 1172, 124-129.

Hutzler, F., Fuchs, I., Gagl, B., Schuster, S., Richlan, F., Braun, M., \& Hawelka, S. (2013). Parafoveal X-masks interfere with foveal word recognition: evidence from fixationrelated brain potentials. Frontiers in systems neuroscience, 7, 33.

Ip, C., Wang, H., \& Fu, S. (2017). Relative expertise affects N170 during selective attention to superimposed face-character images. Psychophysiology, 54, 955- 968.

Itier, R. J., Latinus, M., \& Taylor, M. J. (2006). Face, eye and object early processing: What is the face specificity? NeuroImage, 29, 667-676.

Itier, R. J., \& Taylor, M. J. (2004). N170 or N1? Spatiotemporal differences between object and face processing using ERPs. Cerebral Cortex, 14(2), 132-142.

Jacques, C., d'Arripe, O., \& Rossion, B. (2007). The time course of the inversion effect during individual face discrimination. Journal of vision, 78,3 .

Jacques C. \& Rossion B. (2004). Concurrent processing reveals competition between visual representations of faces. Neuroreport, 15, 2417-2421.

Jemel, B., Pisani, M., Calabria, M., Crommelinck , M., \& Bruyer, R. (2003). Is the N170 for faces cognitively penetrable? Evidence from repetition priming of Mooney faces of familiar and unfamiliar persons. Cognitive Brain Research, 17, 431 - 446. 
Kamienkowski, J.E., Ison, M.J., Quiroga, R.Q., \& Sigman, M. (2012). Fixation-related potentials in visual search: a combined EEG and eye tracking study. Journal of Vision, 12(7), 4.

Kanwisher, N., \& Yovel, G. (2006). The fusiform face area: A cortical region specialized for the perception of faces. Philosophical Transactions of the Royal Society of London B: Biological Science, 361(1476), 2109-2128.

Kauffmann, L., Peyrin, C., Chauvin, A., Entzmann, L., Breuil, C., et al. (2019). Face perception influences the programming of eye movements. Scientific Reports, Nature Publishing Group, 9 (1).

Kaunitz, L.N., Kamienkowski, J.E., Varatharajah, A., Sigman, M., Quiroga, R.Q., \& Ison, M.J. (2014). Looking for a face in the crowd: Fixation-related potentials in an eyemovement visual search task. NeuroImage, 89, 297-305.

Kinoshita, S., \& Hunt, L. (2008). RT distribution analysis of category congruence effects with masked primes. Memory and Cognition. 36, 1324-1334.

Kornrumpf, B., Niefind, F., Sommer, W., \& Dimigen, O. (2016). Neural correlates of word recognition: A systematic comparison of natural reading and rapid serial visual presentation. Journal of Cognitive Neuroscience, 28, 1374-1391.

Kovács, P., Knakker, B., Hermann, P., Kovács, G. \& Vidnyanszky, Z. (2017). Face inversion reveals holistic processing of peripheral faces. Cortex. 97, 81-95.

Kovács, G., Zimmer, M., Bankó, É., Harza, I., Antal, A., \& Vidnyánszky, Z. (2006). Electrophysiological correlates of visual adaptation to faces and body parts in humans. Cerebral Cortex, 16(5), 742-53.

Kovács, G., Zimmer, M., Harza, I., \& Vidnyánszky, Z. (2007). Adaptation duration affects the spatial selectivity of facial aftereffects. Vision Research 47, 3141-3149. 
Langton, S.R.H, Law, A.S., Burton, A.M., \& Schweinberger, S.R. (2008). Attention capture by faces. Cognition, 107 (1). pp. 330-342.

Lavie, N., Ro, T., and Russell, C. (2003). The role of perceptual load in processing distractor faces. Psychological Science, 14, 510-515.

Linkenkaer-Hansen, K., Palva, J.M., Sams, M., Hietanen, J.K., Aronen H.J., \& Ilmoniemi, R.J. (1998). Face-selective processing in human extrastriate cortex around $120 \mathrm{~ms}$ after stimulus onset revealed by magneto- and electroencephalography. Neuroscience Letters, 253, 147-150.

López-Peréz P. J., Dampuré J., Hernández-Cabrera J. A., \& Barber H. A. (2016). Semantic parafoveal-on-foveal effects and preview benefits in reading: Evidence from fixation related potentials. Brain and Language, 162, 29-34.

Lundqvist, D., Flykt, A., \& Öhman, A. (1998). The Karolinska Directed Emotional Faces KDEF, CD ROM from Department of Clinical Neuroscience, Psychology section, Karolinska Institutet, ISBN 91-630-7164-9.

Mares, I., Smith, M. L., Johnson, M. H., \& Senju, A. (2016). Direct gaze facilitates rapid orienting to faces: Evidence from express saccades and saccadic potentials. Biological Psychology, 121(Pt A), 84-90.

Maurer, U., Rossion, B., \& McCandliss, B. D. (2008). Category specificity in early perception: face and word n170 responses differ in both lateralization and habituation properties. Frontiers in human neuroscience, 2, 18.

McKone, E., \& Yovel, G. (2009). Why does picture-plane inversion sometimes dissociate perception of features and spacing in faces, and sometimes not? Toward a new theory of holistic processing. Psychon. Bull. Rev. 16, 778-797

Näasänen, R. (1999). Spatial frequency bandwidth used in the recognition of facial images. Vision Research, 39, 3824-3833. 
Nemrodov, D., Anderson, T., Preston, F.F., \& Itier, R.J. (2014). Early sensitivity for eyes within faces: a new neuronal account of holistic and featural processing. NeuroImage, 97, 81-94.

Niefind, F., \& Dimigen, O. (2016). Dissociating parafoveal preview benefit and parafoveaon-fovea effects during reading: A combined eye tracking and EEG study. Psychophysiology, 53, 1784-1798.

Ossandón, J.P., Helo, A.V., Montefusco-Siegmund, R., \& Maldonado, P.E. (2010). Superposition model predicts EEG occipital activity during free viewing of natural scenes. Journal of Neuroscience, 30, 4787-4795.

Palermo, R., \& Rhodes, G. (2007). Are you always on my mind? A review of how face perception and attention interact. Neuropsychologia, 45, 75-92.

Rama, P., \& Baccino, T. (2010). Eye fixation related potentials (efrps) during object identification. Visual Neuroscience, 27(5-6), 187-192.

Richler, J. J., Mack, M. L., Palmeri, T. J., \& Gauthier, I. (2011). Inverted faces are (eventually) processed holistically. Vision Research. 51, 333-342.

Rossion, B., \& Caharel, S. (2011). ERP evidence for the speed of face categorization in the human brain: Disentangling the contribution of low-level visual cues from face perception. Vision Research, 51, 1297-1311.

Rossion, B., Delvenne, J.-F., Debatisse, D., Goffaux, V., Bruyer, R., Crommelinck, M., \& Guerit, J.-M. (1999). Spatio-temporal localization of the face inversion effect: an event-related potentials study. Biological Psychology, 50(3), 173-189.

Rossion, B., Gauthier, I., Tarr, M. J., Despland, P., Bruyer, R., Linotte, S., \& Crommelinck, M. (2000). The N170 occipito-temporal component is delayed and enhanced to inverted faces but not to inverted objects: An electrophysiological account of facespecific processes in the human brain. NeuroReport, 11(1), 69-74. 
Rossion, B., \& Jacques, C. (2007). Does physical interstimulus variance account for early electrophysiological face sensitive responses in the human brain? Ten lessons on the N170. Neuroimage, 39(4), 1959-1579.

Rossion, B., \& Jacques, C. (2010). The N170: understanding the time course of face perception in the human brain. In The Oxford Handbook of ERP Components, S. Luck and E. Kappenman, eds (Oxford University Press, Oxford, UK).

Rossion, B., Kung, C. C., \& Tarr, M. J. (2004). Visual expertise with nonface objects leads to competition with the early perceptual processing of faces in the human occipitotemporal cortex. Proceedings of the National Academy of Sciences of the United States of America, 101(40), 14521-14526.

Rousselet, G.A., Husk, J.S., Bennett, P. J., \& Sekuler, A. B. (2005). Spatial scaling factors explain eccentricity effects on face ERPs. Journal of Vision, 5, 755-763.

Rousselet, G.A., Pernet, C.R., Bennett, P.J., Sekuler, A.B. (2008). Parametric study of EEG sensitivity to phase noise during face processing. BMC Neuroscience, 9, 98.

Sagiv, N., \& Bentin, S. (2001). Structural encoding of human and schematic faces: holistic and part-based processes. J. Cogn. Neurosci. 13, 937-951.

Schweinberger, S.R., \& Neumann, M.F. (2016). Repetition effects in human ERPs to faces. Cortex, 80, 141-153.

Schweinberger, S.R., Huddy, V., \& Burton, A.M. (2004). N250r: A face-selective brain response to stimulus repetitions. Neuroreport, 15, 1501-1505.

Schweinberger, S.R., Pfutze, E.M., \& Sommer, W. (1995). Repetition priming and associative priming of face recognition — evidence from event-related potentials. Journal of Experimental Psycholoy: Learning, Memory and Cognition, 21 , 722-736. 
Schweinberger, S.R., Pickering, E.C., Jentzsch, I., Burton, A.M., \& Kaufmann, J.M. (2002). Event-related brain potential evidence for a response of inferior temporal cortex to familiar face repetitions . Cognitive Brain Research, 14 , 398 - 409.

Sergent , J. (1984). An investigation into component and configural processes underlying face perception. British Journal of Psychology ,75, 221-242.

Simola, J., Fevre, K.L., Torniainen, J., \& Baccino, T. (2015). Affective processing in natural scene viewing: Valence and arousal interactions in eye-fixation-related potentials. NeuroImage, 106, 21-33.

Soto, V., Tyson-Carr, J., Kokmotou, K., Roberts, H., Cook, S., Fallon, N., Giesbrecht, T., \& Stancak, A. (2018). Brain Responses to Emotional Faces in Natural Settings: A Wireless Mobile EEG Recording Study. Frontiers in psychology, 9, 2003.

Strasburger H., Rentschler I., Juttner M. (2011). Peripheral vision and pattern recognition: A review. Journal of Vision, 11:1-82.

Tanaka, J. W., \& Farah, M. J. (1993). Parts and wholes in face recognition. Quarterly Journal of Experimental Psychology, 46A, 225-245.

Tanaka, J.W., \& Simonyi, D. (2016). The "parts and wholes" of face recognition: A review of the literature. Q. J. Exp. Psychol, ;69:1876-1889.

Tanaka, J., \& Curran, T. (2001). A Neural Basis for Expert Object Recognition. Psychological Science, 12(1), 43-47.

Thierry, G., Martin, C.D., Downing, P., \& Pegna, A.J., (2007). Controlling for interstimulus perceptual variance abolishes N170 face selectivity. Nat. Neurosci. 10, 505-511.

Tottenham, N., Borscheid, A., Ellertsen, K., Marcus, D. J., \& Nelson, C. A. (2002). Categorization of facial expressions in children and adults: Establishing a larger stimulus set. Journal of Cognitive Neuroscience, 14 (Suppl.), S74. 
Vizioli, L., Rousselet, G.A., \& Caldara, R. (2010). Neural repetition suppression to identity is abolished by other-race faces. Proceedings of the National Academy of Sciences of the United States of America, 107(46), 20081-20086.

Yarbus, A.F. (1967). Eye movements and vision. New York: Plenum Press. 